\title{
Effect of Anesthesia on The Outcome of High-Grade Glioma Patients Undergoing Supratentorial Resection: Study Protocol for A Randomized Controlled Trial
}

\section{Dexiang Wang}

Beijing Tiantan Hospital

Jia Dong

Beijing Tiantan Hospital

Min Zeng

Beijing Tiantan Hospital

Xiaoyuan Liu

Beijing Tiantan Hospital

\section{Xiang Yan}

Capital Medical University

\section{Ruowen Li}

Capital Medical University

\section{Shu Li}

Beijing Tiantan Hospital

Yuming Peng ( $\sim$ florapym766@163.com )

Beijing Tiantan Hospital, Capital Medical University https://orcid.org/0000-0002-2630-2467

\section{Ruquan Han}

Beijing Tiantan Hospital

\section{Study protocol}

Keywords: high-grade glioma, anesthesia, survival.

Posted Date: March 10th, 2021

DOl: https://doi.org/10.21203/rs.3.rs-290224/v1

License: (c) (1) This work is licensed under a Creative Commons Attribution 4.0 International License. Read Full License 


\section{Abstract}

Background High-grade glioma (HGG) is the most malignant brain tumor with poor outcome. Whether anesthetic methods have impact on the outcome of these patients is still unknown. Retrospective study has found that there is no difference between two anesthesia methods on the overall survival (OS), however, intravenous anesthesia with propofol might be beneficial in subgroup patients of KPS $<80$. Further prospective studies are needed to evaluate the results.

Methods This is a single-centered, randomized controlled, parallel group trial. 196 patients with primary HGG for tumor resection will be randomly assigned to receive either the intravenous anesthesia with propofol or inhalation anesthesia with sevoflurane. The primary outcome is the OS within 18 months. Secondary outcomes include progression-free survival (PFS), the numerical rating scale (NRS) of pain intensity and sleep quality, the postoperative encephaloedema volume, complications, the length and cost effectiveness of hospital stay of the patients.

Discussion This is a randomized controlled trial to compare the effect of intravenous or inhalation anesthesia maintenance on the outcome of supratentorial HGG patients. The results will help to optimizing the anesthesia methods in these patients.

Trial registration: ClinicalTrials.gov (ID: NCT02756312). Registered on 27 April 2020 https://register.clinicaltrials.gov/

\section{Background}

High-grade glioma (HGG) accounts for $80 \%$ of malignant brain tumor. It is classified by the World Health Organization (WHO) as grade III or IV tumors, and develops the worst prognosis among the brain tumors[1, 2]. Most of HGG patients are glioblastoma patients, with the median overall survival (OS) of 16 months, and the progression-free survival (PFS) ranged from 7 to 10 months even under the optimized treatment $[3,4]$.

Up to now, surgery is still the first-choice treatment to reduce tumor burden and intracranial pressure (ICP). However, most of inhalation anesthesics and the opioids during surgery were regarded as immunosuppressive and enhancing the invasiveness of the tumor cells by preclinical studies. The inhalation anesthesics were found to inhibit natural killer (NK) cell activity [5, 6], and up regulate the level of hypoxia inducible factor (HIF), thus promoting tumor cell proliferation and metastasis, while intravenous anesthesia with propofol can reduce the level of HIF[7]. In vitro study has found that the expression of HIF increased after $6 \mathrm{~h}$ exposure to $2 \%$ sevoflurane in astrocytes ${ }^{[8]}$. On the contrary, propofol promoted the programmed apoptosis of glioblastoma and astrocytoma cells $[9,10]$. Propofol can also reduce the neutrophil-lymphocyte ratio (NLR) and platelet-lymphocyte ratio (PLR), taking protective antiinflammatory effect[11]. All of these are expected to be further verified in long-term outcome of the patients in prospective clinical studies. 
In the retrospective study of 294 patients with supratentorial HGGs undergoing elective tumor resection, anesthesia maintenance with sevoflurane did not significantly worsen the PFS of the patients compared with propofol. However, sevoflurane might decrease the OS compared with propofol in patients with Karnofsky Performance Scale (KPS) score less than 80 [12]. The KPS which is commonly used to evaluate the neurological function in neurosurgery, seemed to affect the association of anesthetics with survival. Several reasons might be related with the results. Firstly, sevoflurane increases cerebral blood flow and ICP, and reduces the compliance of brain tissue, which aggravates the neurofunction of the lower KPS patients [13]. Secondly, compared with sevoflurane, propofol reduces the release of inflammatory factors and vascular permeability, which may reduce the severity of encephaloedema, and contribute to the complete resection of tumor and the early recovery of patients[14]. Lastly, the different impact between propofol and sevoflurane on perioperative period is unclear. Propofol improves postoperative analgesia and promotes rapid recovery compared with inhalational anesthesia[15, 16$]$. So propofol was recommended for better ICP control and cerebral hemodynamics in neurosurgical anesthesia[17, 18]. In the retrospective study, these lower KPS patients mainly included the aged, weak or paralyzed patients. The anesthesia methods might affect the long-term outcome through aggravating the postoperative neurofuntion and systematic condition of patients, however, their effects on tumor malignancy, invasiveness and host cellular immunity have not been identified.

Although the opioids are used perioperatively to block most of the pain or noxious stimulations, they also have negative effect on the cellular immunity and outcome of the patients to some extent[19]. Local anesthetics by scalp infiltration or never block reduce pain effectively, save the use of opioids and inhalation anesthetics, inhibit the growth of tumor cells, protect the patient immunity and the integrity of endothelial cell, and enhance the sensitivity of chemotherapy drugs, so as to improve the prognosis of patients. Zheng et al. has proved that scalp block for glioblastoma surgery was associated with lower inflammatory scores and improved survival[20]. Sung has suggested that the scalp block improved the recurrence profiles of patients receiving primary glioma resection[21]. However, in most of retrospective studies on association between anesthesia and the outcome of tumor patients, the pain intensity, depth of anesthesia and consumption of the anesthetic or analgesic agents were not monitored or controlled comparably and might be a confounder to the results[22, 23]. Although some retrospective studies about other tumor/cancer patients have shown that anesthesia affect patients' outcome, but it is still controversial. An analysis of 7030 patients found that inhalation anesthesia significantly increased the risk of death compared with intravenous anesthesia, with a hazard ratio of about 1.46 [24]. Jun et al. found that inhalation anesthesia with isoflurane, sevoflurane or desflurane increased the risk of recurrence and death of patients compared with intravenous anesthesia in the esophageal cancer patients [25]. Propofol can significantly improve the survival of colon cancer patients compared with desflurane anesthesia [26], however, it did not improve the prognosis of non-small cell lung cancer patients compared with inhalation anesthesia[27]. The retrospective collection of clinical data, unstratified American Society of Anesthesiologists (ASA) classification, tumor pathology and surgery type are all limitations of those studies. A recent systemic review and meta-analysis summarized all previous 
studies by subgroup of cancer source, and indicated that intravenous anesthesia had a certain advantage in general, but not sure in specific tumor source[28].

Therefore, the randomized-controlled trial will be carried out to compare the effect of intravenous and inhalation anesthesia on the 18-months overall survival in patients with supratentorial high-grade glioma. We will test the hypothesis that intravenous anesthesia with propofol could increase the OS and improve neurological function in those patients undergoing tumor resection comparing with sevoflurane maintenance.

\section{Methods And Materials}

\section{Study design}

This is a single-center, randomized-controlled, and paralleled-group trial being conducted at Beijing

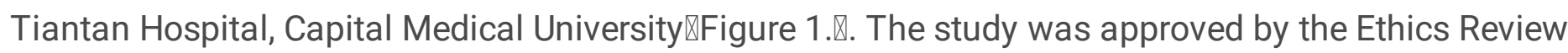
Committee of China Registered Clinical Trials on March 30, 2020 (number: ChiECRCT-20200049) and updated at www.clinicaltrials.gov on April 1, 2020 (number: NCT02756312). The schedule of enrollment and assessment is shown in the Standard Protocol Items: Recommendations for Interventional Trials (SPIRIT) (see Figure 2). Written inform consent will be obtained from the legal representatives. Additional consent will be provided for collection and use of participant data and biological specimens in ancillary studies.

\section{Study population}

\section{Participants}

Patients undergoing selective tumor resection under general anesthesia will be recruited consecutively from neurosurgical wards in Beijing Tiantan Hospital, Capital Medical University from Sept 2020 to December 2023.

Inclusion criteria includes age more than 18 and less than 80 years old, preoperative KPS less than 80, and diagnosis of supratentorial high-grade glioma by preoperative magnetic radiology imaging (MRI). The patients or their relatives agree to provide for collection and use of participant data and biological specimens and sign written informed consent.

Exclusion criteria includes history of other operations after diagnosis of HGG, patients with recurrence and metastasis of gliomas or with malignant tumors of other organs, emergency surgery, ASA physical status of $\mathrm{V}$ or higher, pregnant or breastfeeding women, allergic to study drugs, patients who need electrophysiological monitoring during operation, or patients receiving reoperation with different 
anesthesia methods will be removed. The patients will be withdrawn from the study when postoperative pathological results excluded high-grade glioma.

\section{Randomization and blinding}

Block randomization will be conducted via a computer- generated codes by an independent research assistant who will pack the allocation sequence with identical opaque envelopes and distribute to the researcher according to the randomization. Patients will be randomly assigned to the two groups at a 1:1 ratio. The attending anesthesiologists will perform anesthesia and record intraoperative information. Trained follow-up personnel will not participate in the clinical anesthesia and postoperative management, or know the records of other researchers. The researcher assistant, patients and outcome assessors will be blinded to the allocation until the completion of the study analysis unless specific circumstances, such as the occurrence of a serious adverse event (SAE).

\section{Intervention}

The enrolled patients will be randomly assigned to the intravenous group or the inhalation group. Propofol will be used for anesthesia maintenance with the initial dose of $5-7 \mathrm{mg} / \mathrm{kg} / \mathrm{h}$ in intravenous group. Sevoflurane will be used for anesthesia maintenance with the initial concentration of $2-3 \%$ in inhalation group. During anesthesia, the same depth of anesthesia will be maintained by adjusting the dosage of propofol or sevoflurane, keeping bispectral index (BIS) range between 40 and 60 . Inhalation of sevoflurane, or infusion of propofol and remifentanil will be discontinued at the end of surgery. The adherence of intervention will be guaranteed by the common and routine method of anesthesia maintenance administered in the trial.

\section{Concomitant treatment}

Routine monitoring will include electrocardiograph, noninvasive blood pressure, pulse oxygen saturation, body temperature and BIS. The BIS electrodes will be placed on the contralateral side to the tumor. Core temperature will be monitored continuously by an esophageal probe. Peripheral venous access will be established before anesthesia induction. Continuous arterial pressure, urine output and end-tidal carbon dioxide partial pressure $\left(\mathrm{PetCO}_{2}\right)$, minimal alveolar concentration (MAC) of inhalation agent will be monitored after anesthesia induction. All patients will be premedicated with midazolam $(0.05 \mathrm{mg} / \mathrm{kg})$ intravenously 5 minutes before anesthesia induction. Anesthesia will be induced with propofol (1-3 $\mathrm{mg} / \mathrm{kg})$, sufentanil ( 0.3 to $0.4 \mu \mathrm{g} / \mathrm{kg})$, and rocuronium $(0.6 \mathrm{mg} / \mathrm{kg})$ or cisatracurium $(0.2 \mathrm{mg} / \mathrm{kg})$. After endotracheal intubation, mechanical ventilation will be performed, with a tidal volume 6-8 $\mathrm{ml} / \mathrm{kg}$, a respiratory rate of $12-15 / \mathrm{min}$, a $50-70 \%$ fraction of inspired oxygen in the air and fresh gas at a flow rate of 1-2 L/min to maintain the $\mathrm{PetCO}_{2}$ between 35 and $40 \mathrm{mmHg}$. 
Before skin incision, $0.1-0.2 \mu \mathrm{g} / \mathrm{kg}$ of sufentanil will be given once, and the dosage of remifentanil and sufentanil will be adjusted according to the signs of blood pressure and heart rate. Local anesthetics infiltration in the scalp will be performed at the time of incision. Muscle relaxants can be added intermittently. Crystal or colloid fluid, the urine volume and blood loss will be monitored. Blood transfusion practice guidelines will be resort to once considering blood transfusion[29]. If necessary, vasoactive drugs can be used perioperatively. Single dose of sufentanil $1 \mu \mathrm{g} / \mathrm{kg}$, tramadol $100 \mathrm{mg}$ and ondersetron $8 \mathrm{mg}$ will be given to the patients as dura closure. The patients will be extubated after full recovery from anesthesia and transferred to the post-anesthesia care unit (PACU). The patient-controlled intravenous analgesia will be connected to the patients with sufentanil $100 \mu \mathrm{g}$ and ondansetron $16 \mathrm{mg}$ in $100 \mathrm{ml} 0.9 \%$ sodium chloride solution, with background dose $2 \mathrm{ml} / \mathrm{h}$. After pressing the pump button to reduce pain, one single $0.5 \mathrm{ml}(0.5 \mu \mathrm{g})$ will be infused with 15 minutes locking time.

\section{Outcomes}

The primary outcome is OS (by months) within 18 months postoperatively. OS is the duration from the day of surgery to the date of death or last follow-up. The assessment of outcomes will be performed by trained research assessors who are blinded to the group allocation. Researchers assess the OS through telephone interview patients or their relatives every 3 months postoperatively.

Secondary outcomes include:

1. PFS is the duration from the surgery day to the day of first evidence of progression or death within postoperative-18 month. PFS will be evaluated by using the latest-two MRI tests and clinical evidence according to the RANO (response assessment in neuro-oncology) criteria (Supplementary Table 1). [30]

2. Pain: numerical rating scale (NRS) of pain intensity and sleep quality on postoperative 1 to 3 days will be evaluated. Pain intensity score in NRS is 0 to 10,0 representing no pain and 10 representing worst pain imaginable. Sleep quality with the NRS ( $0=$ worst sleep, $10=$ best sleep $)$ will be used to evaluate the sleep quality at 8 to 10 a.m. every day postoperatively before discharge and every 3 months after discharge.

3. Encephaloedema volume will be calculated from MRI assessed by radiologist at 3 to 7 day postoperatively.

4. Adverse events or complications will be recorded, including congestive heart failure, arrhythmia, myocardial ischemia or infarction, pulmonary edema, pulmonary embolism, respiratory failure, stroke, encephaloedema, hydrocephalus, pneumocranium, intracranial hematomas or infection, epilepsy, gastrointestinal hemorrhage, liver and kidney dysfunction, etc. (Supplementary Table 2, 3)

5. The length and cost effectiveness of hospital stay will be calculated and analyzed.

6. Blood sample will be collected before anesthesia, at the end of surgery and 1 to 3 days after the resection. 


\section{Data collection and management}

After obtaining informed consent, an independent research assistant will initiate baseline information collection one day before surgery. All personal information will be kept strictly confidential for research purposes only. Basic demographic information, including gender, age, vital signs, body mass index, past medical history, family history, smoking and drinking history, allergy history, operation history or anesthesia history, medication history, age-adjusted Charlson comorbidity index, ASA classification, KPS, Glasgow coma scale (GCS) will be collected. Functional classification of tumor (indicating the resectability of tumor) will be evaluated according to the results of preoperative MRI (Supplementary Table 4.) [31,32].

Intraoperative viable include vital sign parameters during anesthesia, total dose or infusion rate of anesthetic agents or other drugs during operation, such as inotropic or vasoactive drugs, estimated blood loss, urine volume, the type and dose of fluid and blood transfusion, duration of surgery and anesthesia. The usage of analgesia pump (dosage, number of compressions and effective compressions) will be recorded.

Postoperative medication will be recorded including sedative, analgesia, glucocorticoids, mannitol and fluid infusion on 1 to 3 day postoperatively. The extent of resection (EOR) will be evaluated by a professional neuro-radiologist according to the results of MRI within 72 hours postoperatively (Supplementary Table 5).

The median follow-up time will be 18 months before the study was terminated. The start points of simultaneous radiotherapy and chemotherapy, patient compliance, adverse events or complications and treatment, the European organization for the research and treatment of cancer on quality of life (EORTCQLQ-C30) and the Karnofsky performance status (KPS) will be evaluated every 3 months postoperatively. Strict data management will be kept including double data entry and establishing range checks for data values.

\section{Sample size calculation and statistical analysis}

The sample size is estimated by using PASS 2011 software (NCCS LLC). We calculated the sample size based on the primary outcome. A two-sided Log-rank test with an overall sample size of 196 subjects (98 in intravenous group and 98 in inhalation group) achieves $80.2 \%$ power at a 0.05 significance level to detect a hazard ratio of 1.66 when the proportion surviving is $62.3 \%$ and $39.2 \%$ in intravenous and inhalation group, respectively. The study will last for about 36 months, of which subject accrual (entry) occurs at the first 18 months with the proportion dropping out of the two group is 0.1 , respectively.

Analysis will be conducted using SPSS software (version 23.0). The Kolmogorov-Smirnov test will be used to test data normality. Normally distributed continuous variables will be described as the means with standard deviation (SD) and compared using independent $t$ tests. Non-normally distributed variables 
will be summarized as medians and interquartile range (IQR) and compared by using Mann-Whitney $U$ tests. Categorical variables will be described as numbers ( $\mathrm{n}$ ) and percentage (\%) and compared using Chisquare or Fisher's exact tests.

The Kaplan-Meier method will be used to describe the time-to-event data (OS and PFS), and the log-rank test will be used for comparisons. Univariate and multivariate Cox proportional hazard regressions will be used for survival analysis. Together with the anesthesia type, variables with $\mathrm{P}<0.1$ in the univariate analysis will be regarded as candidate variables for the multivariable model. A significance level of $\mathrm{P}<0.05$ will be used to indicate statistical significance. The imputation method will be used for dealing with missing data. The impact of missing data will be estimated by sensitivity analysis. Additionally, subgroup analysis will be undertaken to assess the association between anesthesia and the outcome according to the aCCl, KPS, EOR, WHO grade, duration of anesthesia and whether chemotherapy or not. No interim analysis will be done or the trial will not early terminate.

\section{Reporting of adverse events}

Data monitoring committee is not set up in the trial for the sample size is not very large. However, auditing trial will be conducted twice per year and the process will be independent from investigators and the sponsor. All adverse events (AEs) will be closely monitored until a stable situation has been reached. The chief investigator will be informed of any serious AEs and determine the severity and causality of these events. All AEs associated with this study will be recorded and reported to the Ethics Committee as part of the annual report. The chief investigator will be responsible for collecting the details about causes of AEs, treatment, prognosis, and reporting SAE to the Ethics Committee immediately. The incidence of adverse effects will be described as percentage and frequencies for each group. Chi-square and Fisher's exact test will be used for comparison between groups. The researcher will have access to the final trial data set.

\section{Protocol Amendment}

The chief investigator will be responsible for any decision to amend the protocol. If there is any modification (e.g., changes to eligibility criteria, outcomes or analyses), the principle investigator will report and gain approval from the China Ethics Committee of Registering Clinical Trials prior to implementation, by communicating with relevant other members (e.g., investigators, participants, registries, journals, and regulators).

\section{Discussion}

This is a randomized controlled trial to compare the effect of intravenous or inhalation anesthesia maintenance on the OS in supratentorial HGG patients of KPS $<80$. There are some well-known risk 
factors such as age at diagnosis, KPS, histological type, distant metastasis, and extent of surgical resection for predicting the OS of patients with $\mathrm{HGG}[31]$.

Intravenous and inhalation anesthesia are the common choices for HGG resection. But they are different in some aspects. Firstly, intravenous anesthesia inhibit the invasion of the tumor cells and host stress response, and protect the cellular immunity, whilst inhalation anesthesia has the opposite effect. Secondly, during craniotomy, the mass effect of tumor and the peritumor edema always lead to severe intracranial hypertension and brain herniation. As nearly all inhalational agents increase the cerebral blood flow and ICP, propofol has the opposite effect to decrease the ICP and facilitate tumor exposure. Thirdly, intravenous anesthesia promotes postoperative rapid recovery of the patients. Postoperative pain, nausea and vomiting, and shivering appears less frequently with propofol-maintained anesthesia [16, 33, 34]. However, the hemodynamic stability, time to extubation, and early cognitive functions were similar with two anesthetic techniques[35].

In this RCT study, we will fully be evaluated the function of patients before operation, including physical status, neurological function and imaging results. During the operation, standard anesthesia management, anesthesia depth monitoring, individualized medication according to the protocol, and biological samples collection and analysis will be conducted by special trained personnel. After surgery, the patients will be followed up regularly by designated personnel for the recovery, adverse events, complications, treatment, the PFS and OS.

Although the intervention of our study is intravenous or inhalational anesthesia, inhalational anesthetics will not be used for induction, as it is not routine in clinical practice. We regard the effect of single bolus of propofol as minimal and short, which will be eliminated completely before surgical incision. So propofol induction is same in both groups.

Our study will optimized the ideal anesthesia regimen for patients undergoing brain tumor resections, not only from the perioperative anesthesia and analgesia, but also from the point of view on clinical outcomes of overall survival in HGG patients.

\section{Abbreviations}

HGG: High-grade glioma; OS: overall survival; PFS: progression-free survival; NRS: the numerical rating scale; WHO: World Health Organization; ICP: intracranial pressure; NK: natural killer; HIF: hypoxia inducible factor; NLR: neutrophil-lymphocyte ratio; PLR: platelet-lymphocyte ratio; KPS: Karnofsky Performance Scale; ASA: American Society of Anesthesiologists; MRI: magnetic radiology imaging; SAE: serious adverse event; $\mathrm{BIS}$ : bispectral index; $\mathrm{PetCO}_{2}$ : end-tidal carbon dioxide partial pressure; MAC: minimal alveolar concentration; PACU: post-anesthesia care unit. GCS: Glasgow coma scale; EORTCQLQC30: the European organization for the research and treatment of cancer on quality of life; Rano: response assessment in neuro-oncology. 


\section{Declarations}

Trial status: The trial was registered at ClinicalTrials.gov on 29 April 2016 (identifier NCT02756312) and last update time of the trial is 9 Sep 2020. The study was approved by the Ethics Review Committee of China Registered Clinical Trials (Ethics Review No. ChiECRCT-20200049). The trial have not yet recruited any participants till now. The anticipated completion date will be in December 2022.

Ethics approval and consent to participate: The study was approved by Ethics Review Committee of China Registered Clinical Trials (Ethics Review No. ChiECRCT-20200049). All potential risks will be fully explained to the participants or their guardians. The written informed consent will be obtained before enrollment.

Consent for publication: Not applicable.

Availability of data and materials: This study will be conserved in a secure repository at Beijing Tiantan Hospital. The data sets will be available from the chief investigator upon reasonable request.

Competing interests: The authors declare that they have no competing interests.

Funding: This work was supported by Beijing Municipal Administration of Hospitals of Ascent Plan (grant number: DFL20180502). The funding body will not participate in the design of the study and collection, analysis, and interpretation of data.

Author Contributions: DXW, JD, MZ, XYL, XY, RWL, SL, YMP, RQH: conceived the study, contributed to the study design and analytical plans. JD: drafted the protocol. All authors read and approved the final protocol.

Acknowledgements: The authors would like to express their sincere gratitude to the colleagues of the Department of Anesthesiology and the Department of Neurosurgery, Beijing Tiantan Hospital, for their support and cooperation. The authors also would like to express their sincere gratitude to patient advisers for their cooperation in completing the trial. The results of the trial will submit to the academic journal and the abstract will be used to communicate with peer experts in international meetings.

\section{References}

1. Wen PY, Kesari S. Malignant gliomas in adults. N Engl J Med. 2008;359(5):492-507.

2. Louis DN, Ohgaki H, Wiestler OD, Cavenee WK, Burger PC, Jouvet A, Scheithauer BW, Kleihues P. The 2007 who classification of tumours of the central nervous system. Acta Neuropathol. 2007;114(2):97-109.

3. Chinot OL, Wick W, Mason W, Henriksson R, Saran F, Nishikawa R, Carpentier AF, Hoang-Xuan K, Kavan P, Cernea D, Brandes AA, et al. Bevacizumab plus radiotherapy-temozolomide for newly diagnosed glioblastoma. N Engl J Med. 2014;370(8):709-22. 
4. Lamborn KR, Yung WK, Chang SM, Wen PY, Cloughesy TF, DeAngelis LM, Robins HI, Lieberman FS, Fine HA, Fink KL, Junck L, et al. Progression-free survival: An important end point in evaluating therapy for recurrent high-grade gliomas. Neuro Oncol. 2008;10(2):162-70.

5. Buckley A, McQuaid S, Johnson P, Buggy DJ. Effect of anaesthetic technique on the natural killer cell anti-tumour activity of serum from women undergoing breast cancer surgery: A pilot study. Br J Anaesth. 2014;113(Suppl 1):i56-62.

6. Looney M, Doran P, Buggy DJ. Effect of anesthetic technique on serum vascular endothelial growth factor $\mathrm{c}$ and transforming growth factor beta in women undergoing anesthesia and surgery for breast cancer. Anesthesiology. 2010;113(5):1118-25.

7. Luo X, Zhao H, Hennah L, Ning J, Liu J, Tu H, Ma D. Impact of isoflurane on malignant capability of ovarian cancer in vitro. Br J Anaesth. 2015;114(5):831-9.

8. Shi QY, Zhang SJ, Liu L, Chen QS, Yu LN, Zhang FJ, Yan M. Sevoflurane promotes the expansion of glioma stem cells through activation of hypoxia-inducible factors in vitro. $\mathrm{Br} \mathrm{J}$ Anaesth. 2015;114(5):825-30.

9. Hsu SS, Jan CR, Liang WZ. Evaluation of cytotoxicity of propofol and its related mechanism in glioblastoma cells and astrocytes. Environ Toxicol. 2017;32(12):2440-54.

10. Xu J, Xu W, Zhu J. Propofol suppresses proliferation and invasion of glioma cells by upregulating microrna-218 expression. Mol Med Rep. 2015;12(4):4815-20.

11. Ni Eochagain A, Burns D, Riedel B, Sessler DI, Buggy DJ. The effect of anaesthetic technique during primary breast cancer surgery on neutrophil-lymphocyte ratio, platelet-lymphocyte ratio and return to intended oncological therapy. Anaesthesia. 2018;73(5):603-11.

12. Dong J, Zeng M, Ji N, Hao S, Zhou Y, Gao Z, Gu H, Zhang L, Ma D, Peng Y, Han R. Impact of anesthesia on long-term outcomes in patients with supratentorial high-grade glioma undergoing tumor resection: A retrospective cohort. J Neurosurg Anesthesiol. 2020 Jul;32(3):227-233.

13. Conti A, lacopino DG, Fodale V, Micalizzi S, Penna O, Santamaria LB. Cerebral haemodynamic changes during propofol-remifentanil or sevoflurane anaesthesia: Transcranial doppler study under bispectral index monitoring. Br J Anaesth. 2006;97(3):333-9.

14. Yang WC, Zhou LJ, Zhang R, Yue ZY, Dong H, Song CY, Qian H, Lu SJ, Chang FF. Effects of propofol and sevoflurane on aquaporin- 4 and aquaporin- 9 expression in patients performed gliomas resection. Brain Res 2015; 1622:1-6.

15. Qiu QCS, Wong SSC. Effects of intra-operative maintenance of general anaesthesia with propofol on postoperative pain outcomes - a systematic review and meta-analysis. Anaesthesia. 2016;71(10):1222-33.

16. Peng K, Liu HY, Wu SR, Liu H, Zhang ZC, Ji FH. Does propofol anesthesia lead to less postoperative pain compared with inhalational anesthesia?: A systematic review and meta-analysis. Anesth Analg. 2016;123(4):846-58.

17. Chui J, Mariappan R, Fau - Mehta J, Mehta J, Fau - Manninen P, Manninen P, Fau - Venkatraghavan L, Venkatraghavan L. Comparison of propofol and volatile agents for maintenance of anesthesia 
during elective craniotomy procedures: Systematic review and meta-analysis. 1496-8975 (Electronic).

18. Gruenbaum SE, Meng L, Bliotta F. Recent trends in the anesthetic management of craniotomy for supratentorial tumor resection. Curr Opin Anaesthesiol. 2016;29(5):552-7.

19. Plein LM, Rittner HL. Opioids and the immune system - friend or foe. British journal of pharmacology. 2018;175(14):2717-25.

20. Zheng L, Hagan KB, Villarreal J, Keerty V, Chen J, Cata JP. Scalp block for glioblastoma surgery is associated with lower inflammatory scores and improved survival. Minerva Anestesiol. 2017;83(11):1137-45.

21. Sung $\mathrm{CH}$, Tsuang FY, Shih CC, Chang JL, Liao MH, Yang YW, Lee TS, Cheng HL, Wu CY. Scalp block is associated with improved recurrence profiles in patients undergoing primary glioma resection surgery. Journal of neurosurgical anesthesiology.2019.

22. Leslie K, Short TG. Anesthetic depth and long-term survival: An update. Can J Anaesth. 2016;63(2):233-40.

23. Short TG, Leslie K, Campbell D, Chan MT, Corcoran T, O'Loughlin E, Frampton C, Myles P. A pilot study for a prospective, randomized, double-blind trial of the influence of anesthetic depth on long-term outcome. Anesth Analg. 2014;118(5):981-6.

24. Wigmore TJ, Mohammed K, Jhanji S. Long-term survival for patients undergoing volatile versus iv anesthesia for cancer surgery: A retrospective analysis. Anesthesiology. 2016;124(1):69-79.

25. Jun IJ, Jo JY, Kim JI, Chin JH, Kim WJ, Kim HR, Lee EH, Choi IC. Impact of anesthetic agents on overall and recurrence-free survival in patients undergoing esophageal cancer surgery: A retrospective observational study. Sci Rep. 2017;7(1):14020.

26. Wu ZF, Lee MS, Wong CS, Lu CH, Huang YS, Lin KT, Lou YS, Lin C, Chang YC, Lai HC. Propofol-based total intravenous anesthesia is associated with better survival than desflurane anesthesia in colon cancer surgery. Anesthesiology. 2018;129(5):932-41.

27. Oh TK, Kim K, Jheon S, Lee J, Do SH, Hwang JW, Song IA. Long-term oncologic outcomes for patients undergoing volatile versus intravenous anesthesia for non-small cell lung cancer surgery: A retrospective propensity matching analysis. Cancer Control. 2018;25(1):1073274818775360.

28. Yap A, Lopez-Olivo MA, Dubowitz J, Hiller J, Riedel B. Anesthetic technique and cancer outcomes: A meta-analysis of total intravenous versus volatile anesthesia. Can J Anaesth. 2019;4(10):019-330.

29. Practice guidelines for perioperative blood transfusion and adjuvant therapies: An updated report by the american society of anesthesiologists task force on perioperative blood transfusion and adjuvant therapies. Anesthesiology. 2006; 105(1):198-208.

30. Vogelbaum MA, Jost S, Aghi MK, Heimberger AB, Sampson JH, Wen PY, Macdonald DR, Van den Bent MJ, Chang SM. Application of novel response/progression measures for surgically delivered therapies for gliomas: Response assessment in neuro-oncology (rano) working group. Neurosurgery. 2012;70(1):234-43. 
31. Sawaya R, Hammoud M, Schoppa D, Hess KR, Wu SZ, Shi WM, Wildrick DM. Neurosurgical outcomes in a modern series of 400 craniotomies for treatment of parenchymal tumors. Neurosurgery. 1998;42(5):1044-55.

32. Robin AM, Walbert T, Mikkelsen T, Kalkanis SN, Rock J, Lee I, Rosenblum ML. Through the patient's eyes: The value of a comprehensive brain tumor center. J Neurooncol. 2014;119(3):465-72.

33. Citerio G, Pesenti A, Latini R, Masson S, Barlera S, Gaspari F, Franzosi MG. A multicentre, randomised, open-label, controlled trial evaluating equivalence of inhalational and intravenous anaesthesia during elective craniotomy. Eur J Anaesthesiol. 2012;29(8):371-9.

34. Chui J, Mariappan R, Mehta J, Manninen P, Venkatraghavan L. Comparison of propofol and volatile agents for maintenance of anesthesia during elective craniotomy procedures: Systematic review and meta-analysis. Can J Anaesth. 2014;61(4):347-56.

35. Ayrian E, Kaye AD, Varner CL, Guerra C, Vadivelu N, Urman RD, Zelman V, Lumb PD, Rosa G, Bilotta F. Effects of anesthetic management on early postoperative recovery, hemodynamics and pain after supratentorial craniotomy. J Clin Med Res. 2015;7(10):731-41.

\section{Figures}

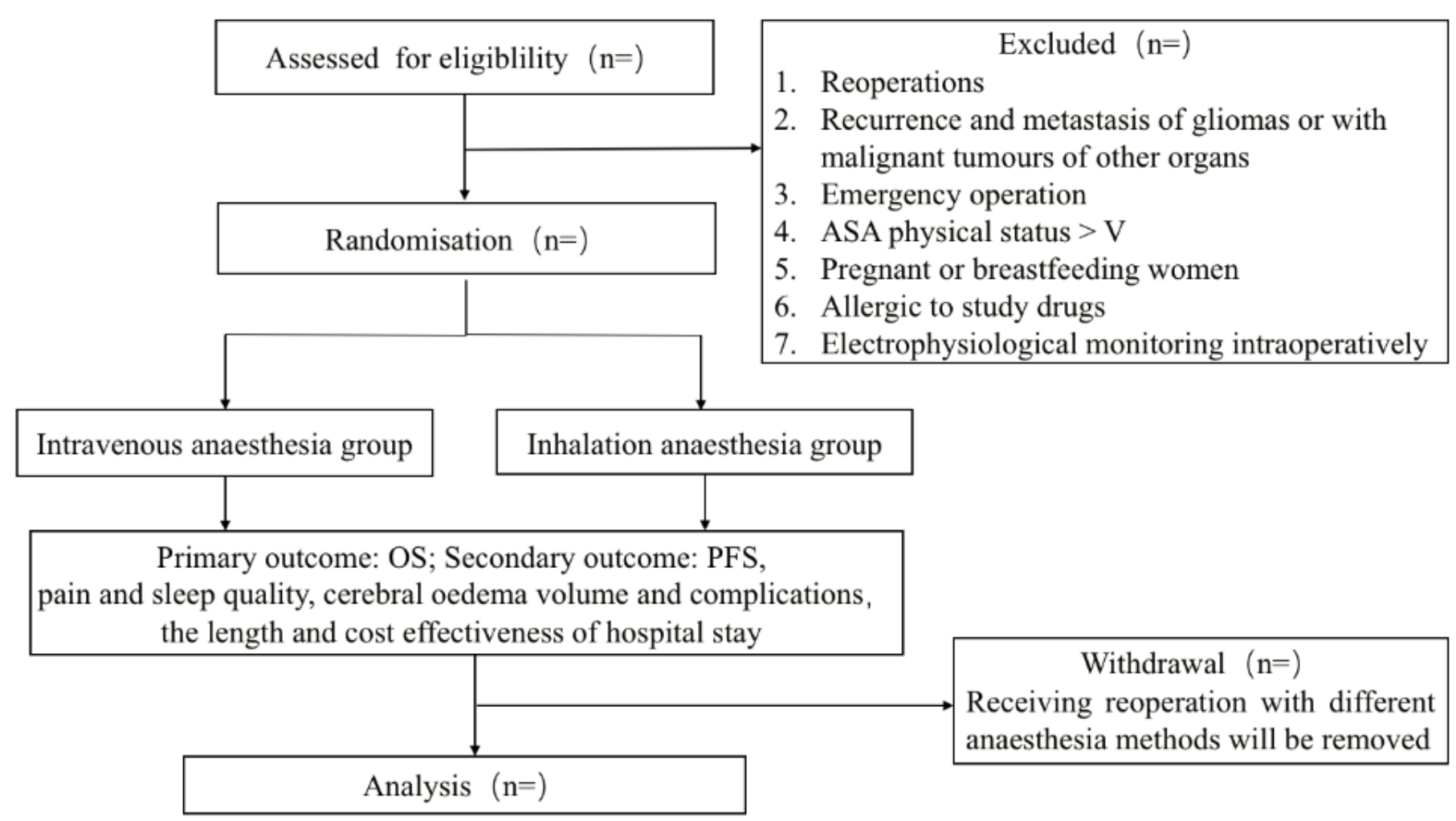

\section{Figure 1}

OS, overall survival; PFS, progression-free survival. 


\begin{tabular}{|c|c|c|c|c|c|c|c|c|c|c|c|c|c|}
\hline \multicolumn{14}{|c|}{ STUDY PERIOD } \\
\hline \multirow{3}{*}{ TIMEPOINT } & \multirow{3}{*}{$\begin{array}{l}\text { Enrollment } \\
\text {-1day }\end{array}$} & \multirow{3}{*}{$\begin{array}{l}\text { Allocation } \\
\text { Sugery day }\end{array}$} & \multicolumn{11}{|c|}{ Post-allocation } \\
\hline & & & \multicolumn{5}{|c|}{ Post-craniotomy(day) } & \multicolumn{6}{|c|}{ Post-craniotomy(month) } \\
\hline & & & 1 & 2 & 3 & 7 & Discharged & 3 & 6 & 9 & 12 & 15 & 18 \\
\hline \multicolumn{14}{|c|}{ ENROLLMENT } \\
\hline Eligibility screen & $x$ & & & & & & & & & & & & \\
\hline Informed consent & $\mathrm{x}$ & & & & & & & & & & & & \\
\hline Allocation & & $x$ & & & & & & & & & & & \\
\hline \multicolumn{14}{|c|}{ INTERVENTIONS } \\
\hline $\begin{array}{l}\text { Inhalation } \\
\text { anesthesia group }\end{array}$ & & $x$ & & & & & & & & & & & \\
\hline $\begin{array}{l}\text { Intravenous } \\
\text { anesthesia group }\end{array}$ & & $\mathrm{x}$ & & & & & & & & & & & \\
\hline \multicolumn{14}{|c|}{ ASSESSMENTS } \\
\hline Baseline variables & & $x$ & & & & & & & & & & & \\
\hline Intraoperative data & & $x$ & & & & & & & & & & & \\
\hline Pain intensity & $x$ & & $x$ & $x$ & $x$ & $x$ & $x$ & $x$ & $x$ & $x$ & $x$ & $x$ & $x$ \\
\hline Sleep quality & $x$ & & $x$ & $x$ & $x$ & $x$ & $x$ & $x$ & $x$ & $x$ & $x$ & $x$ & $x$ \\
\hline GCS & $x$ & & & & $x$ & $x$ & $x$ & $x$ & $x$ & $x$ & $x$ & $x$ & $x$ \\
\hline KPS & $x$ & & & & $x$ & $x$ & $x$ & $x$ & $x$ & $x$ & $x$ & $x$ & $x$ \\
\hline EORTCQLQ-C30 & $x$ & & & & & & $x$ & $x$ & $x$ & $x$ & $x$ & $x$ & $x$ \\
\hline $\begin{array}{l}\text { Encephaloedema } \\
\text { volume }\end{array}$ & $x$ & & & & & & $x$ & & & & & & \\
\hline Adverse Event & & & $x$ & $x$ & $x$ & $x$ & $x$ & $x$ & $x$ & $x$ & $x$ & $x$ & $x$ \\
\hline Tumor sample & & $x$ & & & & & & & & & & & \\
\hline Blood sample & & $x$ & $x$ & $x$ & $x$ & & & & & & & & \\
\hline PFS & & & & & & & & $x$ & $x$ & $x$ & $x$ & $x$ & $x$ \\
\hline os & & & & & & & & $x$ & $x$ & $x$ & $x$ & $x$ & $x$ \\
\hline
\end{tabular}

GCS, Glasgow coma scale; KPS, Karnofsky performance status; EORTCQLQ-C30, European organization for the research and treatment of cancer on quality of life; PFS, progression-free survival; OS, overall survival.

\section{Figure 2}

Standard Protocol Items: Recommendations for Interventional Trials. STUDY

\section{Supplementary Files}

This is a list of supplementary files associated with this preprint. Click to download. 
- SPIRIT0120.doc

- SupplementaryTable.docx 